\title{
Unlearning in crisis: Forces of change in unlearning
}

\author{
Julee Hafner \\ TCSOPP \\ jhhrsearch@gmail.com
}

\begin{abstract}
The study of unlearning continues to be important, not only due to the relevance of the concept itself, but in light of current strong, unforeseen forces, knowledge change opportunities have been created beyond our prediction. A knowledge exchange is often needed to revise processes, use new technologies, or due to forces that stem from catastrophic situations. Examples include economic, such as in business failures or the recent public health concerns from the COVID-19 pandemic. Building from new insights using the typological model from Rushmer and Davies (2004), deep unlearning may the end result of catastrophic forces of change. First, deep unlearning occurs with striking events, or yield change that adds anxiety, psychological, or technological upset. Second, inherent in many catastrophic changes are rapid interruptions in the trajectory of "previous" actions and unique processes toward recovery where knowledge base may be forever altered. We address the following question: "Is Rushmer and Davies' deep unlearning typology exhibited during catastrophic situations?" This theoretical paper examines the concept of deep unlearning, the process of replacement or lack of use of a belief, action, or process in a context of an emergency situation where little is currently known. What type of agent for change would be needed? Will unintended consequences not be identified by individuals and organizations; what may be the cost to future learning skills when deep unlearning of current tasks occurs? Third, some insights and directions for future research are presented.
\end{abstract}

\section{Introduction}

When considering an organizations' ability innovate and forge new directions, strategic formulation of knowledge is analyzed, used, and implemented appropriately for the greatest result and benefit for the organization. However, when reviewing problems such as an organizational bankruptcy, failure to innovate, failure to be aware of the potential for demise, charting the loss of market share or financial decline, leaders fail to focus on the needed issues for change. Their lack of ability to predict current environmental risks and their inability to unlearn past strategies may be a causal reality. Blame may stem from a variety of market changes, staffing, or lack of competent analysis for misperceptions, or a lack of recommended action in time, for problems to be avoided. Often when any crisis occurs, it is imperative that it is first recognized so that a potential change may occur. Whether knowledge is determined as an opportunity, or added threat, can be difficult. A variety of factors need to be identified and processed by leaders including, how the leader evaluates the pool of knowledge, and how it is assessed and perceived by the organization. And finally, how the individual that needs to use the knowledge to make decisions understands the information at hand. All of these factors, even if given enough time are difficult at best. In a pressure situation where time may not be available creates additional considerations.

Failure to notice and heed warnings, may allow improperly adhered to, outdated goals, and beliefs reducing the ability to unlearn. It is in time of rapid change that unlearning may be forced to occur without provocation. According to Darling et al, (2005),

"The constant existence of crises has also taught the world of business that a crisis can occur with little to no warning, anywhere, anytime." [1, p. 344).

When understanding the process of unlearning, three related questions need to be asked: 1) Have the perceptions of knowledge been unlearned differently in an emergency situation versus during a routine context by distorting people's understanding of their internal knowledge during this rapid change? And, 2) Does the process of knowledge change occur differently when there are outside forces driving change? And finally, 3) What can researchers gain from understanding unlearning during this type of situation?

Organizations and individuals have been dealing with unique challenges as a result of unforeseen events named as the novel coronavirus disease in 2019 and later became a pandemic (COVID-19, also 2019-nCoV); subsequently, major changes within all facets of "normal" life have occurred in the United States [2]. Since January 2020, The World Health Organization (WHO) declared a Public Health Emergency with information on the disease outbreak on the new virus, (2019-nCoV) [2].

Almost overnight, countries all over the world rapidly implemented measures aimed at reducing infection rates. From the way individuals chose to behave to the way organizations do business, communicate and interact with 
others, change has been evident. Healthcare and other first responders were challenged with daily experiences of knowledge and practice change in their routine practice.

During the COVID-19 crisis, individuals were immediately forced into restrictive environments, most people had to cope with the restrictions of the "lockdown", creating perceptions of fear, insecurity, and isolation [3]. As a result of the COVID-19 pandemic, organizations were unable to sustain their current established business models and needed to make major shifts in actions, behaviors, and mental models [4]. Practioners were creating new trajectories of care including new infection control practices, treatment of virus-infected individuals, and protecting co-workers from transmission.

Although shifts in business opportunities are often seen during marketplace change on a routine basis, it is evident that there is something different and unique about this particular shift in the type and level of knowledge change currently occurring [1]. There has not been a comparable event in recent memory where so many individuals were faced with uncertainty, inconsistencies in knowledge and rapid change in current knowledge use and practices affecting businesses, workers' health, infection transmission and effective provision of care of its' citizenry.

Marketplace change may make a product or service obsolete requiring the organization or service provider to unlearn. For example, when organizations like Blockbuster, Blackberry, Kodak, Toyota, and Sears no longer supported customers in the way they previously did, they needed to either unlearn what to do, produce and serve differently, or cease operations. History notes a variety of examples of the inability to release current knowledge until incontrovertible new knowledge was presented and not able to be ignored [5]. And even then, many firms failed to do so. This suggests that whether the organization or individual employee was able to accept a new paradigm was key. This process can be facilitated through a trial-and-error process [6].

However, threats to organizations enough to create immediate demise occur rarely in an organizational life span, and has not noted to have occur in recent memory across all daily life. Thus, people and organizations have had no similar experiences or knowledge systems in place to affect these types of change. And, with the speed of impact of a new variety of health concern, change is considered rapidly and forced upon all.

Innovators may need to unlearn who they are by choosing new business models and provide new services for a changing consumer [5]. This normal process has happened since the days of the horse - drawn carriage and the buggy whip with both being examples that involved routine change. Organizations are required to use updated information to maintain competitive advantage and remain viable. However, when change is considered rapid and disruptive, organizations and individuals have to deal with unintended and unforeseen consequences.

Reaction to massive and widespread change may become swift and painful with perceptions of anxiety and fear. Change of this nature, created by an outbreak of a viral illness creates change for organizations, health department practioners and individuals as they are not immune to the possibility of infection themselves [3]. Inconsistent practioner knowledge adds to the perception of uncertainty. Anecdotal evidence is easy to come by when speaking to any healthcare provider, such as the author. The COVID-19 pandemic demonstrates how change can be swift and pervasive [5]. Continual change occurred on a daily basis.

It can be difficult to prepare for and envision how to acquire knowledge, transmit knowledge, and to create the needed changes to reduce the personal and organizational impact. When there is a rapid onset of any change, it requires rapid decision making, and rapid action to determine the best course of action should be, and what technological solutions could be [4]. Opportunity in a markedly different way is needed to avoid technological upset and unintended consequences of unsuccessful knowledge change [6].

To reduce this impact, systemic change through individual unlearning is necessary [7]. Rushmer \& Davies (2004), suggested knowledge change may involve different typologies of unlearning: routine, wiping, and deep unlearning [8]. In previous work, wiping was investigated to determine how unlearning occurs in healthcare organizations and individuals [9]. Although in light of current world conditions, it may be time to revisit this model, and build upon it to understand rapid change using examples from the current COVID-19 pandemic from a theoretical lens. This vantage point along with the author's practical healthcare expertise, may shed insight to problems we currently face in unlearning routines that no longer work for us and how unlearning continues to affect us all.

The present paper focuses on new challenges created by the COVID-19 pandemic and suggests the process for deep unlearning is involved in how healthcare individuals and organizations alike process their previous knowledge base during a crisis situation [8].

With healthcare practioners responsible for maintaining competency and providing error-free service, the strategy of how to successfully update processes or "unlearn" previous actions and produce new competencies has been of interest [7], [8], [9]. Unlearning has been defined previously, as the process of removing, discarding, or eliminating an action, procedure, or belief in favor of a new one [10]. With the ability to acquire and internalize task competencies, especially in healthcare practioners, successful unlearning skills are needed [7], [9]. However, unlearning requires a previously acquired knowledge base of learning, a specific learned familiarity, or competency [7]. Noted use in organizational and individual change processes, ongoing change of knowledge, and of specific competencies are essential to updating information and knowledge in professional practice [7]. Successful unlearning represents the recognition that current knowledge requires updating and actions to begin knowledge change should be initiated [9], [10], [11], [12].

In addition, the process may involve whether the process had finished as in complete unlearning, or the process was 
stalled in some way, as in incomplete unlearning [9]. Without a specific accepted understanding of these differences between complete and incomplete unlearning process, and the situations where unlearning can occur, how to successfully create knowledge change within healthcare practioners will remain unsolved.

Practioners need to understand what change are to be made, and develop competencies. When healthcare organizations require updated competencies, the process is to update previously learned, routinized knowledge. However, the time and method it takes is often unaccounted for and is placed as part of routine change processes when new knowledge becomes available. In light of current world conditions, this process has been streamlined and may yield upset and frustration [6]. Undergoing knowledge change and developing knowledge competencies remains an ongoing problem for healthcare professionals [7], [11], [12].

Whether knowledge is actually discarded and replaced also remains under investigation. How healthcare practioners' previously acquired knowledge base is altered when being updated also is not known. Now, with change occurring rapidly in near-crisis situations, even more is study is needed to facilitate successful unlearning and reduce unintended consequences for organizations and providers.

'Often organizations require a 'forceful trigger' to begin the process of unlearning after a failure or during crisis management" [8, p. 96].

When individual unlearning is not successful, key changes fail to occur. It is the trigger that may be responsible or help to produce initiation of the unlearning process, especially in a crisis situation.

\section{Related Literature}

\subsection{Learning and Unlearning}

Learning involves acquiring new information and processing it into learned responses. With repetition, through practice, the actions become habituated and represent a current knowledge base. This base can be that involves successful performance of the new tasks without errors continues to represent an important focus for healthcare practioners [6].

Literature on the process of unlearning remains limited although it can trace its development of these important concepts to the 1980's [9]. With technological advances in acquisition and use of knowledge and its management, a new interest about unlearning has emerged. Researchers have continued to return to unlearning due to its importance in maintaining competencies and understanding change processes. Understanding the unlearning process may facilitate the technological ease during change of knowledge for employees as it becomes better understood [13].

One key area where unlearning knowledge is particularly limited is in the characteristics of the individual level unlearning process. To remain able in the midst of change requires the employee to acquire, refine, and retain knowledge [14]. The individual's ability to change is essential to avoid crisis when they are the main drivers of organizational activity. During knowledge change, work product errors may impact practioner competency and healthcare service delivery.

Continual learning in healthcare practioners is often considered routine. To overcome rapid changes in quality of care has remained a challenge in light of new emerging crisis situations [3], [5], [8]. When people need to stop their current behavior and begin doing things differently in a rapid manner has been the focus during a crisis such as the COVID-19 pandemic [5], [15]. "Unlearning at the individual level then also includes a deliberate ending of specific routines that involve this particular knowledge" [15, p., 869].

Deciding the age-old question of 'What works?' suggests time, energy, and money is needed to determine the impact of intervention, and the ability to unlearn what does not work [16, p. 255]. With speed being critical, the appropriate change processes are essential.

Unlearning is now required by providers, with the understanding that when they tackle new challenges related to changes on service provision in a crisis situation [17]. The theoretical analysis developed here builds from and extends the work of Rushmer and Davies (2004) which suggest that there are three typological structures that unlearning can be based [8]. This paper aims to conceptualize unlearning, specifically 'deep unlearning" as within the current reality of uncertainty [18].

Unlearning is the process of replacement or disuse of knowledge, action, or procedure substituting new knowledge when appropriate [10]. Through unlearning, previously learned knowledge or procedures are modified by adding emerging skills with new knowledge, thus completing the learning process [19]. Whether the individual has control over change or it is an unconscious activity process remains unaccounted for during unlearning. The present knowledge base and individual learning style impacts learning competency, but it may also impact unlearning.

When individual unlearning is not successful, and employees remain in their position, errors can occur, thus creating increased confusion and tension in the individual [9]. Errors may consist of slow, incorrect, or inconsistent actions. Causal factors of errors may include interruptions in learning behavior or faulty processes during change [12], [14]. Decreased productivity, reduced quality, and additional costs may be unintended consequences of these errors resulting from organizational change [21].

The use of unlearning as part of the process of gaining new knowledge involves total removal of old knowledge [9]. Knowledge acquisition and modification has been speculated to involve "replacement" of prior knowledge [19]. Newstrom (1983) posited individuals begin with a "clean slate" before adding information [22]. This suggests that the brain actually 
erases unneeded information [23]. Clark (2010) discounted this concept, as faulty suggesting knowledge cannot be added to infinitely. This would suggest an ever-expanding brain that stores and processes vast amounts of data [24]. Or, this process may require a reduction of complete acquisition due to "space" requirements to hold the new knowledge [21, p. 59]

However, practioners require the realization that previous knowledge is unreliable and they need to stop using it [5]. Nystrom \& Starbuck (2011, p. 36) suggest that the idea that an individual should "eliminate preexisting knowledge or habits that would otherwise represent formidable barriers to new learning" was suggested, but has not been empirically established [24].

Often viewed as a complex cognitive process, unlearning may be an unrecognized and unused, yet important, part of the learning cycle. However, practioners must unlearn previously used knowledge to as technology advances [20]. Recently acquired knowledge often remains untested by the individual [21]. When knowledge is absorbed, it becomes part of the awareness of the individual, but it is not necessarily used [25]. Acquiring and changing competency from the previous learned knowledge base can be difficult for healthcare practioners resulting in confusions and technological upset while knowledge is tested [9], [11], [26].

Bloom's taxonomy provides additional framework and study foundation. Three domains that relate to knowledge acquisition have been identified that impacts unlearning: the affective, the psychomotor, and the cognitive domain [27]. The affective domain focuses on the way the learner responds to learning. The psychomotor domain focuses on the actions, accuracy, and rate the learner performs the task [27]. Learning of factual knowledge and abilities acquired through recall are present involve the cognitive domain [27].

The difference with unlearning involves mental skill changes with a previously learned knowledge base. How the brain changes old unconscious behaviors, specifically in the area of retrieval and use as well as storage and disuse of into new automatic behaviors, may be a function of the unlearning process. With continual emphasis on unlearning skills to update the old, the process is continual. However, consistency in repetition, knowledge storage and retrieval systems need to be in place, for complete unlearning to occur [28].

Researchers have investigated unlearning from diverse perspectives with an ongoing disagreement about the differences between learning and unlearning. For healthcare practioners, implementation of new technology or processes may result in the need for revisions of current knowledge base and actions to correctly perform updated job functions.

Conforming to numerous procedural and technological changes of employees has been a previous focus, however not specifically in healthcare practioners. The process of successful knowledge use and change requires understanding of how updating in practioners occur [23], [26]. Learning strategies and other teaching methods assist in making modifications needed, but fail to focus on the difficulties some practioners may have completing updated job functions, resulting in an incomplete unlearning process and stress. The following table (Table 1) summarizes some of the major influencers to unlearning theory. From this table, it is noted that unlearning varies per specific lens used.

Before the arrival of a major disruption, the inherent complexity of any change within an organization may be difficult, let alone within a crisis. With a diverse phenomenon, such in as in a healthcare epidemic, many facets require the unique strategy of unlearning that uses a different approach to solve change process problems. Determining what needs to be unlearned is critical to reduce errors and perform successfully, especially in crisis [4]. Knowledge management of the unlearning process should involve understanding of perceptions of deep unlearning to more fully understand the unlearning process in crisis.

\subsection{Rushmer Davies Typologies of Unlearning}

In Rushmer and Davies (2004) typologies, unlearning was explained to demonstrate a differentiation between knowledge change situations. The first typology, 'routine unlearning' may suggest that there is a passive replacement of behavior due to changes in a process or routine [8]. No effort is used to produce change, it may occur over time through disuse of information.

Knowledge change, the second typology, involving updating new procedures and behaviors, called 'wiping', occurs with choice and deliberation of the change. The individual may experiment and reflect upon the change process. The individual is deliberate about no behaving or producing a specific action. They may decide to act or may be influenced to change [8]. What no longer works for them is recognized and something different is undertaken.

Wiping occurs when the impact of new knowledge is strong enough to recognize errors or misconceptions in their current knowledge base and it requires updating. For example, when a new protocol for a drug treatment becomes standardized in healthcare; or in computer systems, when systems are upgraded due to the operation inefficiency are two examples of a wiping typology [8]. Both represent an ability to make a change within a process of behavior when needed. Routine and wiping involve slow transitional changes to the use of new methods. Each unlearning level adds increased rate for actions and how they are initiated.

It is the third typology which is of interest in this discussion. The third typology, or 'deep unlearning', is characterized as disruptive, often including a sudden event occurring with great speed whereby the initiation of unlearning is directed from an outside catalyst [8]. This description from Rushmer and Davies (2004) of a crisis suggests a not only a rapid onset, but a need for solution to the event. The experience is often described as painful and occurs quickly limiting information processing or reflection. Previous routines are no longer the same and complete 


\begin{tabular}{|c|c|c|c|}
\hline Theorist & Year & Type & Related Theory \\
\hline $\begin{array}{l}\text { Akgun, et } \\
\text { al. }\end{array}$ & (1978) & Organizational & Acquired knowledge may be untested. \\
\hline $\begin{array}{l}\text { Argyris \& } \\
\text { Schon }\end{array}$ & $(1977)$ & Individual & $\begin{array}{l}\text { Single loop and double learning to differentiate } \\
\text { action change. }\end{array}$ \\
\hline Bandura & $(1956)$ & Individual & Observation of behavior to create change. \\
\hline $\begin{array}{l}\text { Bloom \& } \\
\text { Krathwohl }\end{array}$ & $(2010)$ & Individual & $\begin{array}{l}\text { Three learning domains: Cognitive, Affective and } \\
\text { Psychomotor. }\end{array}$ \\
\hline Clark & $(2017)$ & Both & $\begin{array}{l}\text { Limited awareness of how they learn; unconscious } \\
\text { knowledge acquisition. }\end{array}$ \\
\hline $\begin{array}{l}\text { Cegarra- } \\
\text { Navarro, } \\
\text { and } \\
\text { Wensley }\end{array}$ & (2019) & Organizational & $\begin{array}{l}\text { Defined intentional unlearning with an } \\
\text { "unlearning cycle" }\end{array}$ \\
\hline Dewey & $(1916)$ & Individual & $\begin{array}{l}\text { Constructivist Learning Theory using the } \\
\text { instructor to provide learning frameworks. }\end{array}$ \\
\hline $\begin{array}{l}\text { Grisold, } \\
\text { Kaiser, and } \\
\text { Hafner }\end{array}$ & $(2019)$ & Organizational & Organizational envisioning for future change \\
\hline $\begin{array}{l}\text { Griswold, } \\
\text { Klammer, } \\
\text { and Kragulj }\end{array}$ & $(2020)$ & Organizational & $\begin{array}{l}\text { Two forms of organizational unlearning; open- } \\
\text { ended unlearning yielding unknown outcomes, } \\
\text { and goal-directed where changes are unique to } \\
\text { previously established knowledge }\end{array}$ \\
\hline $\begin{array}{l}\text { Hislop, } \\
\text { Bosley, and } \\
\text { Coombs }\end{array}$ & $(2017)$ & Both & Current theories in unlearning change \\
\hline $\begin{array}{l}\text { Klammer, } \\
\text { Grisold, and } \\
\text { Ngyuen }\end{array}$ & $(2019 b)$ & Organizational & Perspectives in Organizational change \\
\hline $\begin{array}{l}\text { Neal, Wood, } \\
\text { \& Quinn }\end{array}$ & $(2006)$ & Individual & $\begin{array}{l}\text { Repetition over time produces learning. Obstacles } \\
\text { to learning may exist reducing learning. }\end{array}$ \\
\hline Peschl & (2019) & Organizational & Organizational change in uncertainty \\
\hline $\begin{array}{l}\text { Nonaka \& } \\
\text { Takeuchi }\end{array}$ & (1995) & Organization & $\begin{array}{l}\text { SECI Model: knowledge is used in four ways: } \\
\text { socialization (S- tacit to tacit); external (E- explicit } \\
\text { to explicit); conceptualization (C- tacit to explicit); } \\
\text { internalization (I- explicit to tacit). }\end{array}$ \\
\hline $\begin{array}{l}\text { Quinn, } \\
\text { Pascoe, } \\
\text { Wood, \& } \\
\text { Neal }\end{array}$ & $(2009)$ & Both & $\begin{array}{l}\text { Stimulation in context and repetition produces } \\
\text { learning of a habit }\end{array}$ \\
\hline Senge & $(2006)$ & Both & Mental models are the basis of learning. \\
\hline Skinner & (1953) & Individual & Reinforced behavior produces learning. \\
\hline Starbuck & $(1996)$ & Both & Recognition where knowledge is faulty. \\
\hline
\end{tabular}

Figure 1: Table of

Unlearning Theorists a fire and creating an emergency event where family members run from their home without belongings in order to prevent loss of life. Another example would be the severity of weather conditions, such as tornados, hurricanes, and typhoons where limited planning and immediate actions are needed to survive [1].

Deep unlearning, involving transformational change during a crisis event, and where a unique process of unlearning is used [8]. The main difference between the typologies are the speed of onset, appearance, and characteristics of outside forces that drive the process. Individuals are faced with conditions that may jeopardize life, limb and ongoing survival for the organization or individual. In addition, there is also other components such as stress and emotional fallout, described in technological upset perceptions, reduced task performance accuracy, and other unintended consequences that have been initially identified during the process [13].

Healthcare organizations and practioners must change their actions quickly and effectively to produce new outcomes, especially in time of crisis were successful unlearning needs to occur rapidly to update skill competencies and practices. Completely changing this knowledge base involves the successful alteration and use of this new knowledge however, researchers are uncertain as to the process [26].

To reduce this impact, systemic change through individual unlearning is necessary [28]. The concern about being able to change information rapidly in light of crisis, the disposition of old information, and the ability to override previous learning when needed are difficult for practioners.

Continuing confusion regarding characteristics of unlearning lacks empirical agreement consisting of anecdotal evidence about the process. A review of the literature may consist of many features and process dynamics [25], [26]. Unlearning may be an additional factor to consider during successful knowledge change. Complete unlearning occurs when updated knowledge is incorporated successfully into practioner patient care routines and medical errors eliminated [9].

Although unlearning terminology is now considered multidisciplinary, lack of a consistent definition remains without consensus. Unlearning is a knowledge change process; however, empirical identification of specific factors contributing to completion of the process is unknown.

The nature of a rapid change can provide a new paradigm and offers an updated model of the unlearning change process. As all businesses experience change, good and bad, it is the organization that can understand the dynamics of needed change and when to unlearn. The number of models is numerous and may not represent the complex organizational processes of unlearning in a variety of situations. A brief review and summary Table (Table 1 ) represents transformation has occurred due an action taken [8]. An example might be of a highly combustible substance causing 
many of the major theorists in the field of unlearning and provides the reader with further study options.

Therefore, this paper presents an alternative model during rapid, unforeseen, rapid onset healthcare change. For these discussion purposes, we make use of the COVID-19 pandemic as an example of swift and pervasive change where it can be difficult to envision how to reduce the impact through successful unlearning. Although there have been more examples in industry and history where unlearning had failed to occur, such as WWII attack on Pearl Harbor, The Bay of Pigs incident, the Challenger disaster, and in business such as, Daimler-Chrysler, US- Airlines and Braniff Airlines bankruptcies, Toyota, or British Petroleum, and processes such as PC development at IBM. These examples, although catastrophic to the individual organizations could be foreseen and through proper change processes, some of these organizations are still doing business. We consider the nature of this unique paradigm shift and how change occurs when it is impacted in a rapid manner. It is not the ability to decide failures or successes of the processes, the relationship of homeostasis within an organization or life span of that organization. The sequence of stages is may be defined within updating of Rushmer and Davies' model can define systems where unlearning can be brought about, namely in crisis situations. Therefore, this paper represents an updated, presentation of this theoretical model of the unlearning process in rapid emergency situations and may help organizations develop new processes to make modifications in their systems.

The following paper will shed some light on unlearning in knowledge practioners during crisis. Healthcare practioners require accuracy and competencies even in the most challenging situations to complete their functions. To stay competent, providers must maintain a previous knowledge base as a routine scope of practice. Healthcare organizations may benefit from an ongoing investigation of unlearning especially in crisis situations. With the vast amount of knowledge need to remain a competent healthcare practioner, knowledge base requires continued updating to new competencies. When a situation occurs where time is of the essence, deep unlearning maybe needed. Understanding the complexities of the process of deep unlearning continue be an excellent focus for continued research. Here, we address the following theoretical research question:

“Are Rushmer and Davies' deep unlearning typologies exhibited during crisis by healthcare practioners' in the unlearning process?"

\subsection{Unlearning in Crisis}

In organizations, leaders attempt to make changes that are planned, calculated and drive the organization to success. Some managers may limit the change factors and prevent unlearning because it is difficult to admit that processes are no longer working. To try new ideas, old routines must be discarded. [25].

Why do crises occur, and how do organizations and individuals react? [25] Organizational crisis may be created, and is dependent through the leader's vision, ability to predict change, and evaluation of marketplace conditions. It is also dependent on individual cognitive make-up [25]. Cognition is created through experiences, attitudes and mental models [20].

Darling (2004) suggests that crisis is always a possibility and with the unpredictability during an organization's life cycle, it should be planned to occur [1]. If an emergency situation occurs, it is the organization that defines what constitutes a significant problem, the importance to business longevity, and what type of change and help to produce the change is needed.

Unfortunately, with a rapid change in the health of employees, creating unknown overarching consequences for unintended related problems, acting in a speedy manner does not give the organization to formulate plans and react in light of additional governmental intrusion. Adding to these difficulties, were supply and personnel shortages. Even determining the frequency and amount of exposure, procurement of enough supplies and determining precautions, not to mention the inconsistent perceptions of the employees and in question throughout the weeks where the situation unfolded.

How much can the organization be expected to take control of the knowledge and respond based upon inconsistent, often competing knowledge; how quickly can a firm be expected to correctly act on faulty knowledge? This was the case of a variety of organizations providing healthcare in hospitals, clinics and homes. It is the ability of the practioner to unlearn that will be essential in meeting the ever-changing needs within an organization.

When an organization's survival is in jeopardy, questioning of previously held beliefs and actions may be suspect. Conflicts occur as awareness of unlearning is needed and knowledge routines of processes are challenged. Crisis has been described as an event or situation that can reduce the possibility of an organization's survival; it can also be considered a difficult time where a quick solution is needed and not be available [5], [25]. It is interesting to note that in the Chinese language, the character represented as crisis are the symbols for opportunity and danger.

Acquiring and changing competency from the previous learned knowledge base can be difficult for healthcare practioners creating upset when speed is essential [6], [8], [14]. McInerney and Day (2007) suggested that the learning process in an individual is important to the expression of knowledge and transmission of that knowledge, thus resulting in competency with other organizational individuals [29].

With continual emphasis on unlearning skills to update the old knowledge base, the process is continual. Practioners involved in skill changes must be able to discard their current competencies and mental models in favor of the new knowledge [20]. However, consistent behavioral repetition within a workplace environment is required for successful 
service delivery daily actions [5]. When unlearning is unsuccessful, errors in actions may result. During updating processes where actions are already in a state of flux, such as in updating technology, understanding unlearning may prove useful, especially deriving frameworks from learning theories.

As knowledge changes continually, today's healthcare practioners are faced with the difficult task of keeping pace especially in light of the inconsistent messages and current knowledge about COVID-19 [2]. Implementation of any new process may result in added difficulty to complete a change in processes successfully. Different job functions brought about by catastrophic disruptions have the potential to increase work product errors, not to mention technological upset and other unintended consequences for the practioner [6]. Therefore, technological changes in healthcare create an ongoing need to unlearn old competencies. Without changes to maintain competency, practioners may expend additional time and energy while noting perceptions of strain during crisis change.

"From early in the pandemic, reports from countries around the world have detailed experiences of increased emotional distress, symptoms of depression, anxiety, insomnia and overall mental health disturbances among frontline staff" [31].

In practice, instruments used by healthcare practioners, specifically hearing professionals, are often upgraded with new versions or replaced with new technology to more closely support service delivery functions. Many of the users develop unconscious or rote behavior even when working with new technology [9]. These changes require that practioners and other users continually revise their mental models and processes in using new versions [6].

Bhagavathula, et, al., (2020) surveyed healthcare practioners to determine their perceptions regarding this crisis. Results indicated the need for reduction of stress within healthcare workers was created by the lack of consistent knowledge [32].

For example, when there is a significant gap, and discrepancies in consistent messaging, and inconsistent perceptions of COVID-19, actions are often not able to be unlearned as there is no consistency or stable knowledge to produce repetition of actions, beliefs, or mental models [6], [8], [28]. As the global threat of COVID-19 continued to emerge, knowledge consistency in the provider practioner ws required.

During transformational learning of a new competency, employees use previously acquired knowledge until new knowledge becomes available. To utilize newly acquired knowledge, a realization between old and emerging new skills must occur [7], [9], [26]. The individual then produces change with knowledge processing and stabilization to become automatic actions, and "mental models" through unlearning [7], [11], [12], [14], [19].
What no longer works in a crisis situation, needs to be rapidly identified from what was previously taken-forgranted assumptions. This is often a challenging process because many routines and assumptions, are so embedded in current activity that are often not perceived [4].

The model Figure 1 helps to explain the process of deep unlearning driven by an outside force. For this discussion, the COVID-19 pandemic serves to be the framework for a situation of deep unlearning that needed to occur.

The massive machinery of governments, health organizations and other knowledge-based systems needed to rapidly change in order to save lives and prevent further transmission of the COVID-19 virus [2]. As displayed in Figure 1, rapid changes may produce shock, and an extinguishment of previous actions, skill, competency, habits, and mental models. Healthcare practioners needed to do things differently on a daily basis. Treatments previous help as curative no longer worked, which defined the process of deep unlearning on a practical level. Perceptions of healthcare providers reported shock, upset and even noted that it affected their personal well-being [3]. Organizations needed to respond quickly and attempted to provide their assistance in corporate change and resilience for healthcare workers [5]. Even though attempts were valiant, many workers reported upset and stress at lack of resources and support. There was no time for experimentation central to wiping [8].

Unlearning is effortful and still may be unsuccessful in the best of circumstances. There needs to be a perceived urgency to break routinized patterns; with COVID-19 crisis change became evident. Organizations and individuals need to be focused and committed.

Due to immediacy of the pandemic circumstances not to mention the evolving knowledge and generalized uncertainty, practioners noted characteristics of deep unlearning [31]. Currently, as in this public health instance, information is continuing to evolve, so the techniques used in crisis management may or not prove useful.

The following figure (Figure 1) has been developed to, in theory, display the possible operations of the unlearning process during the current situation.

\subsection{Discussion and need for research}

Many organizations can have unique and diverse ways to address any threat, situation, or crises. How individuals react can mean reduced undesired outcomes and require the process of unlearning. Would the use of crisis management tools, systems, and better communication skill will benefit the healthcare practioner- undoubtedly so, however the speed of impact in any emergency situation requires the initial phase of unlearning to occur, namely awareness.

Organizations now are using new knowledge structures, risk assessment, and other tools found within the crisis management sciences while using current knowledge capital to complete the deep unlearning process such as in the COVID-19 global crisis. 


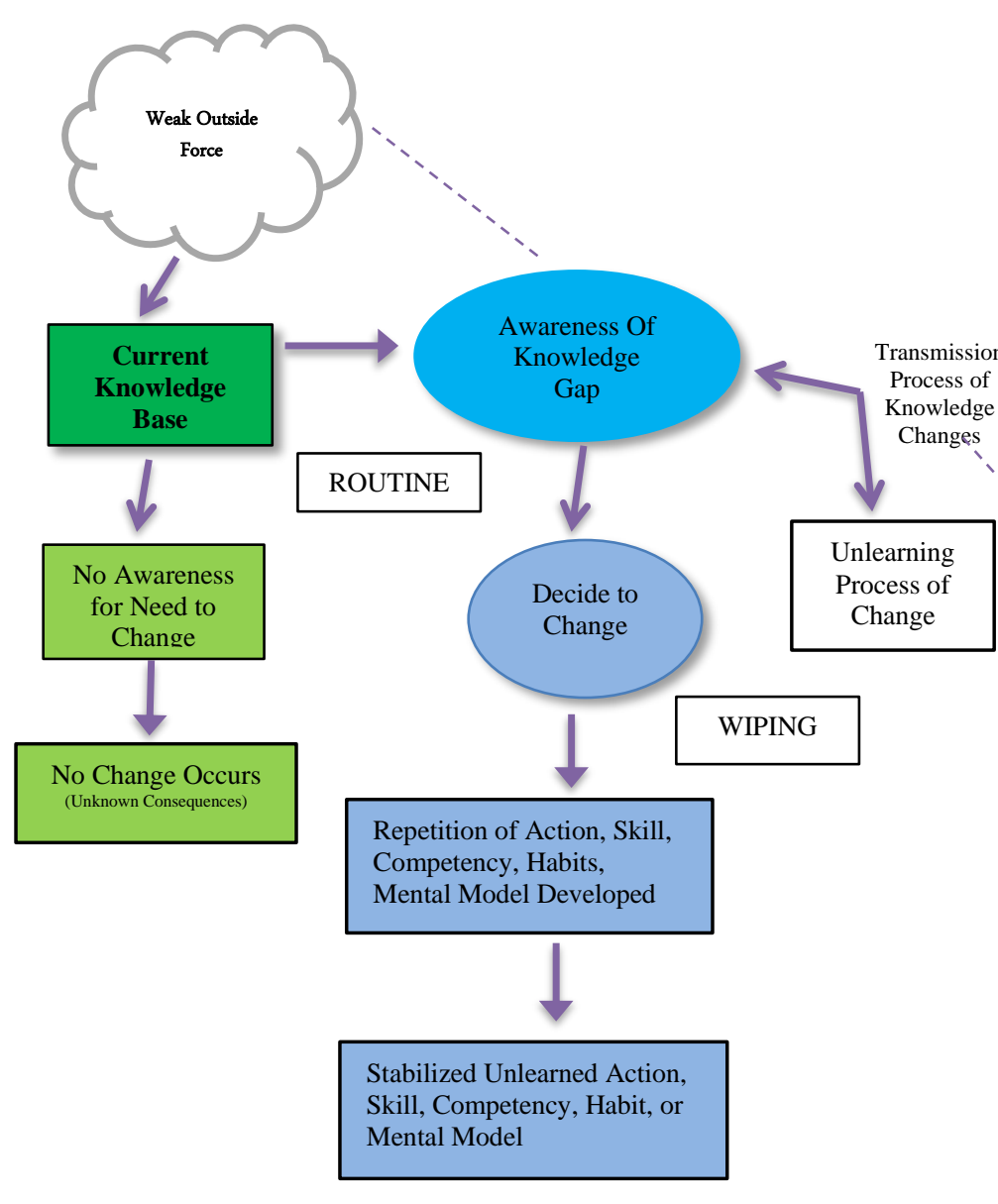

In this Rushmer and Davies' (2004), each situation from an outside force has created the need for deep unlearning. A disruptive, often including a sudden event with the initiation of unlearning directed from an outside catalyst without awareness or warning [7].
And yet, there are many unsolved issues to tackle. When strategies to work through knowledge change is essential, as during COVID-19, understanding and designing knowledge strategies during disruption is needed to complete the process and unlearn. Due to the need for consistent updating, unlearning may play an important role in successful practioner knowledge change during catastrophic changes involved in deep unlearning process seen in a pandemic such as COVID- 19.

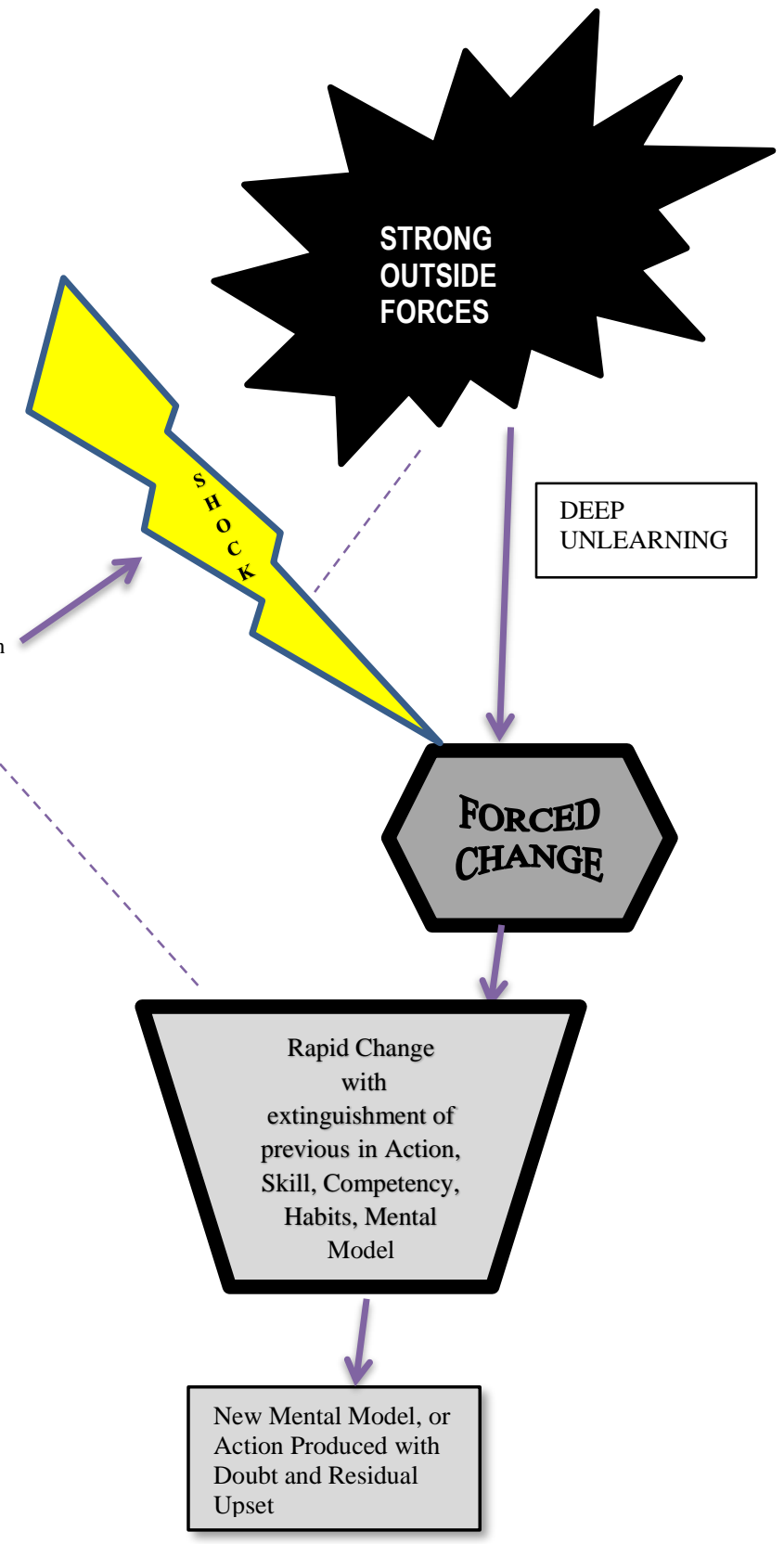

Surviving and recovery may involve the understanding of deep unlearning when a pandemic is officially over [2], [8] in successful practioner knowledge change during catastrophic changes involved in deep unlearning process seen in a pandemic such as COVID- 19.

What can be unlearned during this type of situation? If each employee can identify and prioritize what knowledge is relevant then outdated, and obsolete item can be readied for 
replacement. Because adults create and use a variety of types of knowledge in a variety of processes to manage knowledge, change processes become critical. Facilitating knowledge change successfully and avoiding erred or obsolete knowledge is important to individuals that need to change existing knowledge base for competency requirements.

The first is to develop awareness of possible threats and develop knowledge management systems to create consistent knowledge base. Put mock systems in place and test; in most healthcare organizations disaster drills are routine. Make unlearning a part of drill and required educational practices by charging routines to avoid complacency. This involves the increase and diversity of advice and information for the healthcare organization. Create the williness to support the unlearning of incorrect information and chart a new course when needed; be able to release information that is no longer working. Emphasize successful and complete change processes.

Second, in all problems, unlearning may be triggered through inaction, uncertainty and complacency which may reduce a leaders' vision to issues requiring knowledge change. Failure to manage expertise, innovative opportunities, and time may only add to need for the skill of successful unlearning. There is limited research on how unlearning models describe organizations work to shift between stages, the processes of emergency awareness or how to remove a threat to reduce the impacts of severe change, and none appear in the current literature. Researchers need to use retrospective study to quantify these opportunities for unlearning when managing knowledge.

Third, support the professionals that do the majority of actions during crisis and throughout the unlearning process with consistent training practices. Any type of turnarounds will require needs for additional capital, unlearning education, additional financial resources and the reduction of unprofitable activities so that possible liquidation can be avoided in the organization.

Surviving and recovery may involve the understanding of deep unlearning when a pandemic is officially over and researchers may need to examine this retrospectively in their fields of study. Due to the need for consistent updating, unlearning may play an important role in successful practioner knowledge change during catastrophic changes involved in deep unlearning process seen in a pandemic such as COVID- 19 [2], [8]. Good case study and grounded theory studies can be undertaken for new insights into the unlearning process. This model proposed here may begin to describe the process of unlearning and explain how different crisis knowledge is in that it requires healthcare practioners to develop accuracy and expertise during practice.

Confronted with any disruptive phenomenon, such as public health issues, a realization that old processes and skills no longer work; there needs to remain a recognition that a current gap between previous and current knowledge is present and need to be addressed. And the worker may be undergoing personal stressors that need to be accounted for throughout the process.
This paper adds a perspective to the current practical understanding of the unlearning process within crisis through the use of the Rushmer and Davies model [8]. In deep unlearning, there is may be no time to make assessments; actions are immediately required creating technological upset. New skills are forced to occur and current knowledge base is radically altered.

When an employee can be supported to identify their current knowledge and properly process and prioritize what needs to be unlearned, items can be readied for replacement. Managing manage knowledge in these types of situations where change processes become critical to rapid action and solution remain challenging. Organizations need to facilitate training to change knowledge successfully and avoid confusions in faulty knowledge so practioners can update their existing knowledge base for competency requirements.

The present complexity of the COVID-19 global crisis has yielded many unsolved problems that an understanding of unlearning may provide these solutions. With a new model (Figure 1) of the Rushmer and Davies' (2004) typologies, an updated description of the process of unlearning has been proposed to explain crisis unlearning.

With better understanding of successful knowledge change during the crisis situation of deep unlearning, practioners could avoid unsuccessful unlearning and their unintended consequences. And with increased understanding of deep unlearning processes, organizations may explain and prepare how they can facilitate change in a positive manner, reducing the impact of shock and technological upset for their employees dealing with these types of changes, and survive in an uncertain world.

\subsection{References}

[1] Darling, J., Seristo, H., \& Gabrielsson, M. (2005). Anatomy of crisis management: a case focusing on a major cross-cultural clash within Daimler-Chrysler. Liiketaloudellinen aikakauskirja, 3, 343.

[2] "Novel Coronavirus (2019-nCoV) SITUATION REPORT - 1" (PDF). World Health Organization. 20 January 2020. Retrieved 7 June 2021.

[3] Büssing, A., Rodrigues Recchia, D., Hein, R. et al. Perceived changes of specific attitudes, perceptions and behaviors during the Corona pandemic and their relation to wellbeing. Health Qual Life Outcomes 18, 374 (2020). https://doi.org/10.1186/s12955-020-01623-6

[4] Thomas Grissold interview for Nedeljnik. Need for Unlearning retrieved from: retrieved on 6/2/2021 from: https://ovationbbdo.rs/en/news-en/need-for-unlearning/

[5] Orth, D., \& Schuldis, P. M. (2021). Organizational learning and unlearning capabilities for resilience during COVID-19. Learning Organization.

[6] Hafner, J. H. (2015, January). Computer System Unlearning in Individuals. In System Sciences (HICSS), 2014 48th Hawaii International Conference on (pp. 38603869). IEEE. 6 
[7] Becker, Karen Louise (2007) Unlearning in the workplace : a mixed methods study. PhD thesis, Queensland University of Technology

[8] Rushmer, R., \& Davies, H. T. (2004). Unlearning in health care. Quality and safety in Health Care, 13(suppl II), ii 10- ii 15. doi: 10.1136/qsch. 2003.009506

[9] Hafner, J. (2017). Error Occurrence: Successful versus Unsuccessful Unlearning in Hearing Aid Professionals, 2017 Proceedings of the 50th Hawaii International Conference on Systems Science, Hawaii, 2015 - ieeexplore.ieee.org.

[10] Hedberg, B. (1991), How Organizations Learn and Unlearn. In P. Nystrom \& W. H. Starbuck (Eds.), Handbook of Organizational Design (Vol. 1). London: Cambridge University Press.

[11] Reeler, D., Unlearning- facing up to the real challenge of unlearning. Community Development Resource Association, retrieved on 4.14.12 from http://www.cdra.org.za.

[12] Starbuck, W. H. (1996). Unlearning ineffective or obsolete technologies. International Journal of Technology Management, 11 (7, 8), 725.

[13] Noteboom, C., \& Hafner, J. (2015). Challenges of Change: Technological Ease or Technological Upset?. In Twenty-first Americas Conference on Information Systems, Puerto Rico.

[14] Low, P. K. C., (2011). Must we Unlearn to Learn?. International Research Journals, Vol. (2) pp.1801hhtp://www.interesjournals.org/ER

[15] Klammer, A., \& Gueldenberg, S. (2019). Unlearning and forgetting in organizations: a systematic review of literature. Journal of Knowledge management.

[16] Davies, H., Nutley, S., \& Tilley, N. (2000). Debates on the role of experimentation. What works, 251-276.

[17] Cegarra-Navarro, J. G., \& Wensley, A. (2019). Promoting intentional unlearning through an unlearning cycle. Journal of Organizational Change Management.

[18] Morais-Storz, M. and Nguyen, N. (2017) "The role of unlearning in metamorphosis and strategic resilience", The Learning Organization, Vol. 24 Issue: 2, pp.93-106, https://doi.org/10.1108/TLO-12-2016-0091

[19] Starbuck, W. H. (1996). Unlearning ineffective or obsolete technologies. International Journal of Technology Management, $11(7,8), 725.4$

[20] Senge, P. M. (2006). The fifth discipline: The art and practice of the learning organization. New York: Doubleday

[21] Becker, K. (2008). Unlearning as a driver of sustainable change and innovation: three Australian case studies. International Journal of Technology Management, 42(1), 89-106.

[22] Newstrom, J. W. (1983). The management of unlearning: Exploding the "Clean Slate" fallacy. Training and Development Journal, 37(4), 53.

[23] Clark, R. E. (2010). Cognitive and neuroscience research on learning and instruction: Recent insights about the impact of non-conscious knowledge on problem solving, higher order thinking skills and interactive cyberlearning environments. 11th International Conference on
Education Research (ICER), New Educational Paradigm for Learning and Instruction, Seoul, South Korea, 1-24. Retrieved from http://www. aect. org/publications/whitepapers/2010/ICER3.pdf

[24] Nystrom, P., \& Starbuck, W. H. (2015). To avoid organizational crises, unlearn. Unlearn (December 25, 2015).

[25] Becker, K. (2007). Facilitating unlearning during implementation of new technology. Management, 23(3), 251-268.

[26] Griswold, T. \& Kaiser, A. (2017). Leaving Behind What We are Not: Applying a Systems Thinking Perspective to Present Unlearning as an Enabler for Finding the Best Version of the Self, Journal of Organizational Transformation \& Social Change, 14:1, 39-55, DOI: 10.1080/14779633.2017.1291145

[27] Bloom, B. S., \& Krathwohl, D. R. (1956). Taxonomy of educational objectives: The classification of educational goals (Handbook 1: Cognitive domain). New York, NY: Longmans.

[28] Hislop, D. (2013). Knowledge management in organizations: A critical introduction. Oxford University Press.

[29] McInerney and Day (2007) McInerney, C. R., \& Day, R. E. (2007). Rethinking knowledge management (Vol. 12). Berlin, Germany: Springer-Verlag.

[30] Nonaka, I., and von Krogh, G., (2009). Tacit Knowledge and Knowledge Conversion: Controversy and Advancement in Organizational Knowledge Creation Theory, Organization Science, Vol. 20, No. 3, May-June 2009, p. 635-652.

[31] Parsons Leigh, J., Kemp, L.G., de Grood, C. et, al. A qualitative study of physician perceptions and experiences of caring for critically ill patients in the context of resource strain during the first wave of the COVID-19 pandemic. BMC Health Serv Res 21, $374 \quad$ (2021). https://doi.org/10.1186/s12913-021-06393-5

[32] Bhagavathula, A. S., Aldhaleei, W. A., Rahmani, J., Mahabadi, M. A., \& Bandari, D. K. (2020). Knowledge and Perceptions of COVID-19 Among Health Care Workers: Cross-Sectional Study. JMIR public health and surveillance, 6(2), e19160. https://doi.org/10.2196/19160

[33] Hislop, D, Bosley, S, Coombs, CR, et al. (2014) The process of individual unlearning: A neglected topic in an under-researched field. Management Learning 45(5): 540560.

[34] Grisold, T, Kaiser, A, Hafner, J (2017) Unlearning before creating new knowledge: A cognitive process. In: Proceedings of the fiftieth annual Hawaii international conference on system sciences (HICSS-50), Kohala Coast, HI, 4-7 January. New York: IEEE.

[35] Klammer, A, Grisold, T, Ngyuen, N (2019b) Opportunities and interdisciplinary perspectives for organizational unlearning. The Learning Organization 26(5): 445-453.

[36] Peschl, M. F. (2019) Unlearning towards an uncertain future: On the back end of future-driven unlearning. The Learning Organization 26(5): 454-469. 